\title{
A NOTE ON UNIQUELY MAXIMAL BANACH SPACES
}

\author{
by E. R. COWIE
}

(Received 16th September 1981)

Let $X$ be a real or complex Banach space with norm $\|$.$\| . Let G$ denote the set of all isometric automorphisms on $X$. Then $G$ is a bounded subgroup of the group of all invertible operators $G L(X)$ in $B(X)$. We shall call $G$ the group of isometries with respect to the norm $\|$.$\| . A bounded subgroup of G L(X)$ is said to be maximal if it is not contained in any larger bounded subgroup. The Banach space $X$ has maximal norm if $G$ is maximal. Hilbert spaces have maximal norm. For the (real or complex) spaces $c_{0}, l_{p}$ $(1 \leqq p<\infty), L_{p}[0,1](1 \leqq p<\infty)$, Pelczynski and Rolewicz have shown that the standard norms are maximal ([3], pp. 252-265). In finite dimensional spaces the only maximal groups of isometries are the groups of orthogonal transformations. Given any bounded group $H$ in $B(X), X$ can be renormed equivalently so that each $T \in H$ is an isometry, by $\|x\|_{1}=\sup \{\|T x\| ; T \in H\}$. Therefore corresponding to every maximal subgroup $G$ there is at least one maximal norm for which $G$ is the group of isometries. In this paper we shall investigate those maximal groups $G$ for which there is only one maximal norm with $G$ as its group of isometries.

We have the following definition:

Definition 1. The Banach space $X$ has uniquely maximal norm if it has maximal norm and there is no equivalent norm, not a linear multiple of the original norm, with the same group of isometries.

Uniquely maximal and maximal are not equivalent for norms on a Banach space. Consider the following examples.

Example 2. The standard norm in the real Banach space $l_{1}$ is maximal. The isometries in $l_{1}$ are of the form $U\left(\left\{x_{n}\right\}\right)=\left\{\alpha_{n} x_{\sigma(n)}\right\}$ where $\alpha_{n}= \pm 1$ and $\sigma$ is a permutation ([1], p. 178). Define

$$
\left\|x_{n}\right\|_{0}=\sup \left\{\sum x_{n} y_{n} ;\left|y_{n}\right| \leqq 1,\left|y_{n}-y_{m}\right| \leqq 1,\left|y_{n}+y_{m}\right| \leqq 1\right\}
$$

Then $\|.\|_{0}$ is an equivalent norm on $l_{1}$ with the same group of isometries as the usual norm $\|.\|_{1}$, and is not a linear multiple of the original norm. Hence the standard norm in $l_{1}$ is not uniquely maximal.

Example 3. In [2], Kalton and Wood showed that the uniform norm is a maximal norm for the complex Banach space $C[0,1]$. The isometries in $C[0,1]$ are of the form $U(f)(t)=\alpha(t) f(\phi(t))$ for all $f \in C[0,1], t \in[0,1]$ where $\alpha(t)$ is a continuous function such 
that $|\alpha(t)|=1$ for all $t \in[0,1]$ and $\phi$ is a homeomorphism of $[0,1]$ (see [1], p. 173). Define $\|f\|_{1}=\|f\|+|f(0)|+|f(1)|$ for all $f \in C[0,1]$. Then $\|\cdot\|_{1}$ is an equivalent norm with the same group of isometries but is not a linear multiple of the original norm. Hence the uniform norm on $C[0,1]$ is not uniquely maximal.

We shall now obtain a characterisation of uniquely maximal norms. They turn out to be exactly those norms which are convex transitive. A norm is called convex transitive if $\overline{\mathrm{co}}\{U x ; U \in G\}=\{y ;\|y\| \leqq 1\}$ for each $x \in X$ with $\|x\|=1$.

In order to prove this result we shall require the following lemma.

Lemma 4. Let $X$ have a uniquely maximal norm. Then $\|f\|=\sup \{|f(U x)| ; U \in G\}$ for each $x \in X$ with $\|x\|=1$ and each $f \in X^{*}$.

Proof. Fix $f \in X^{*}$. Define

$$
\|x\|_{1}=\|x\|+\sup \{|f(U x)| ; U \in G\} \text { for all } x \in X .
$$

Then $\|\cdot\|_{1}$ is an equivalent norm on $X$.

If $V \in G$, then

$$
\|V x\|_{1}=\|V x\|+\sup \{|f(U V x)| ; U \in G\}=\|x\|+\sup \{|f(U x)| ; U \in G\}=\|x\|_{1} .
$$

Therefore $\|\cdot\|_{1}$ has at least the same isometries as $\|$.$\| . Hence as the norm is uniquely$ maximal,

$$
\|x\|_{1}=\|x\|+\sup \{|f(U x)| ; U \in G\}=k\|x\|
$$

for all $x \in X$ and some constant $k>0$. We have $\sup \{|f(U x)| ; U \in G\}=r\|x\|$ for all $x \in X$ and some constant $r>0$.

Now $|f(U x)| \leqq\|f\|\|U x\|=\|f\|\|x\|$ for all $U \in G$. Therefore $r \leqq\|f\|$. Given $\varepsilon>0$ there exists $y \in X$ with $\|y\|=1$ such that $|f(y)| \geqq\|f\|-\varepsilon$. Hence

$$
r=\sup \{|f(U y)| ; U \in G\} \geqq|f(y)| \geqq\|f\|-\varepsilon .
$$

We have proved $\quad \sup \{|f(U x)| ; U \in G\}=\|f\|\|x\|$ for all $x \in X$.

Theorem 5. For a Banach space $X$, the norm is uniquely maximal if and only if the norm is convex transitive.

Proof. Assume $X$ has a convex transitive norm. Fix $x \in X$ with $\|x\|=1$. Then $\overline{c o}\{U x$; $U \in G\}=\{y ;\|y\| \leqq 1\}$. Suppose there exists an equivalent norm $\|\cdot\|_{1}$ on $X$ with isometries $G_{1}$ such that $G \subseteq G_{1}$. Let $y \in X$ with $\|y\|=1$. Given $\varepsilon>0$ there exists $\left\{U_{1}, \ldots, U_{n}\right\} \subseteq G$ and $\left\{\lambda_{1}, \ldots, \lambda_{n}\right\} \subseteq \mathbb{P}^{+}$such that

$$
\left\|y-\sum_{1}^{n} \lambda_{m} U_{m}(x)\right\|<\varepsilon, \text { and } \sum_{1}^{n} \lambda_{m}=1 .
$$


We have

$$
\begin{aligned}
\|y\|_{1} & \leqq y-\sum_{1}^{n} \lambda_{m} U_{m}(x)\left\|_{1}+\right\| \sum_{1}^{n} \lambda_{m} U_{m}(x) \|_{1} \\
& \leqq K\left\|y-\sum_{1}^{n} \lambda_{m} U_{m}(x)\right\|+\sum_{1}^{n} \lambda_{m}\left\|U_{m}(x)\right\|_{1} \\
& \leqq K \varepsilon+\|x\|_{1} \text { for some constant } K \text { by equivalence. }
\end{aligned}
$$

Hence $\|y\|_{1} \leqq\|x\|_{1}$, and similarly $\|x\|_{1} \leqq\|y\|_{1}$. Therefore $\{x ;\|x\|=1\} \subseteq\left\{y ;\|y\|_{1}=r\right\}$ for some $r>0$, that is, $r\|x\|=\|x\|_{1}$ for all $x \in X$. Hence the norm is uniquely maximal.

The above proof is essentially the proof given by Rolewicz ([3], p. 256) that a convex transitive norm is a maximal norm.

Suppose that the norm is not convex transitive. Then there exists $x \in X$ with $\|x\|=1$ such that

$$
B=\overline{\mathrm{co}}\{U x ; U \in G\} \varsubsetneqq\{y ;\|y\| \leqq 1\} .
$$

Let $z \in\{y ;\|y\| \leqq 1\} \backslash B$. By the Hahn Banach separation theorem (see [4], p. 60) there exists $f \in X^{*}$, the dual space of $X$, such that $|f(x)| \leqq 1$ for all $x \in B$ and $|f(z)|>1$. But by Lemma $4, B$ is a norming set for $f$, which is a contradiction.

By the results of Rolewicz ([3], $\$ 6$ and 7) on convex transitive norms, the spaces $L_{p}[0,1](1 \leqq p<\infty)$ and the space $C_{0}[0,1]$ (all continuous complex valued functions vanishing at the end points, see Example 2) have uniquely maximal norms.

\section{REFERENCES}

1. BANACH, Theorie des operations lineaires (Warsaw, 1932).

2. N. J. Kalton and G.V. Wood, Orthonormal systems in Banach spaces and their applications, Math. Proc. Camb. Phil. Soc. 79 (1976), 493-510.

3. Rolewicz, Metric linear spaces (PWN, Warsaw, 1972).

4. Rudin, Functional analysis (McGraw-Hill, New York, 1973).

Department of Pure Mathematics

University College of Swansea

Singleton Park

SWANSEA, SA2 8PP 